\title{
More models of muscle movement
}

\author{
Physical models of phenomena such as muscle movement continue to be elaborated, but their relevance to the real \\ world will be clearer only when the structure of the molecules concerned is better known.
}

IT may be that last year's neatest measurement was that of the movement of kinesin molecules along the surfaces of microtubules (K. Svoboda, C.F. Schmidt, B.J. Schnapp \& S.M. Block Nature 365, 721; 1993). Svoboda and his colleagues from the late Edwin Land's Rowland Institute of Science in Cambridge, Massachusetts, showed, by direct optical measurement, that kinesin (like myosin, one of nature's agents for effecting mechanical motion) will, in the presence of ATP, move along a microtubule to which it is attached in consecutive steps about 8.2 ( \pm 1.1) nanometres long, often (in the real cell) dragging a cellular organelle behind it.

The neatness of the measurement, or at least its sophistication, is not questioned. Kinesin molecules were passively anchored to spherical silica beads $0.6 \mu \mathrm{m}$ in diameter, which were then loaded on to microtubules. The essence of the arrangement is a microscope for focusing a split laser beam onto two overlapping spots separated by $250 \mathrm{~nm}$ in such a way that the position of the sphere is at once made visible and constrained, or "trapped", within a region of a few hundred nanometres across. Svoboda and colleagues estimate that the restoring force (towards the centre of the overlapping laser spots) on a silica sphere near the edge of the field amounts to 5 pico-newtons, about enough to lift 0.005 micrograms against gravity.

The measurement is important as well as neat, as Jonathon Howard remarked at the time (Nature 365, 696; 1993). For one thing, the average speed of movement turns out to be a sensitive function of the concentration of ATP; the more ATP, the more jumps a second, from a few to nearly a hundred. Second, and more immediately telling, the estimated size of each step along a microtubule is almost exactly that expected from what is known about the structure of tubulin, the essential ingredient of microtubules, which consists of two similar but distinct proteins anchored together head to tail, and which span just $8 \mathrm{~nm}$.

Polymers of these dimers then associate laterally, perhaps 13 of them at a time, to form mechanically relatively rigid microtubules. The inference is that there is one binding site on each tubulin element. From this it is also possible to infer that each step taken along a microtubule requires the hydrolysis of one molecule of ATP.

As luck will have it, there is not yet much to say about the structure of the active heads of the kinesin molecules except that they are two-headed. But since Svoboda et al. appeared, the mechanisms by which myosin NATURE · VOL 368 - 24 MARCH 1994 molecules effect the relative movement of muscle fibres has been described even more graphically than can be done for kinesin by a further refinement of the same technique (J.T. Finer, R.M. Simmons \& J.A. Spudich Nature 368,$113 ; 1994)$. The hydrolysis of ATP induces a conformational change in the head which then induces movement by mechanical leverage. It is not unreasonable to suppose that something much like this happens with kinesin. The protein attachment of kinesin to a microtubule must change shape when it hydrolyses one molecule of ATP, and the nett effect must be that it moves one $8 \mathrm{~nm}$ step along the microtubule.

But how does the kinesin "know" (anthropomorphically speaking) in which direction it should move? Quite separately from biologists' interests in what cells are up to, there has grown up a minor industry with a bearing on this question. Six months ago, Marcelo Magnasco from the Nippon Electric Company's research laboratory at Princeton and the Rockefeller University in New York described one way in which a linear motor, as in a muscle, might function (Nature 365, 203; 1993), generating the energy needed to sustain a mechanical force "for free", as he put it, from its thermally fluctuating surroundings.

The guiding principle is that of an asymmetrical energy-well which is replicated indefinitely in some direction. It may, for example, be a saw-tooth structure in which the two edges of the teeth make different angles with the vertical. This, then, is a ratchet. Suppose then that there is a particle in one of the potential wells, that the particle itself is in communication with a heat bath at some fixed temperature (and so subject to random energy fluctuations) and that it is pulled in one direction or the other by a fixed force, not by itself sufficient to pull the particle in its own direction.

Magnasco sought to define the conditions in which the particle would move consistently in the opposite direction, against the force, doing work in the process. His conclusion was that if the ratchet is correctly oriented (with the more gently rising slope in the direction of the intended motion) and if the energy fluctuations are not strictly thermal (but "coloured", as they say in the trade), the particle would indeed move against the direction of the external force, doing work in the process, or acting as if it were a mechanical rectifier.

Now R. Dean Astumian and Martin Bier from the University of Chicago have taken the argument a step further (Phys. Rev. Lett.
72, 1766-1769; 14 March 1994). In their view (expressed after the appearance of Svoboda et al.), fluctuations of the forces to which the particle is exposed and fluctuations of the height of the potential barrier may be more realistic than other assumptions. The second model is supported by some speculation about the way in which electrical charges carried by ATP may neutralize the natural charge pattern of the protein substrate (in the case of kinesin, the tubulin polymer filaments).

Certainly the numerical agreement between the outcome of these calculations and the measurements of Svoboda is remarkable. Astumian and Bier also conclude, for example, that at the highest rates of motion of kinesin along a microtubule filament, more than one molecule of ATP may be needed to accomplish each step. On the other hand, the prediction that lower speed might make still more economical use of ATP appears not to be borne out by Svoboda et al.'s observation of the uniform motion of kinesin molecules at low ATP concentrations.

What matters in all this is the degree to which the models are likely to be useful not merely in accommodating the data now certain to be harvested in large quantities, but in suggesting whether some observations are likely to be more pertinent than others. That polymerized tubulin filaments must have the structure of a ratchet seems to go without saying, and accords with the idea that the tubulin dimer consists of two similar proteins joined head-to-tail. That is why, no doubt, it turns out that intact microtubules turn out to be unidirectional.

The obvious gap in the argument is the glaring uncertainty about the function of ATP in real life. If microtubules are ratchets and if the role of ATP hydrolysis is, say, to bring the two heads of kinesin together, it is easy to believe that only the relaxation step (following hydrolysis) will be controlled by the ambient fluctuations, thermal or otherwise. Even so, those embarking on the intricate optical measurement of contractile molecules such as those pioneered by Svoboda et al. could do worse than read the modest literature of the model-builders.

Meanwhile, the objection that the modelbuilders are pushing violations of the second law of thermodynamics should be suppressed. "Coloured" noise is itself a sign of a system out of equilibrium. The interesting question is rather that of how the hydrolysis of ATP by contractile proteins can simulate the appearance of fluctuations that are not boringly Gaussian.

John Maddox 